PROCEEDINGS OF THE

AMERICAN MATHEMATICAL SOCIETY

Volume 137, Number 12, December 2009, Pages 4199-4202

S 0002-9939(09)10048-5

Article electronically published on July 27, 2009

\title{
STABILITY IN $L^{1}$ OF CIRCULAR VORTEX PATCHES
}

\author{
THOMAS C. SIDERIS AND LUIS VEGA
}

(Communicated by Walter Craig)

\begin{abstract}
The motion of incompressible and ideal fluids is studied in the plane. The stability in $L^{1}$ of circular vortex patches is established among the class of all bounded vortex patches of equal strength.
\end{abstract}

For planar incompressible and ideal fluid flow, the theory of Yudovich 9] establishes global well-posedness of the initial value problem with initial vorticity in $L^{1}\left(\mathbb{R}^{2}\right) \cap L^{\infty}\left(\mathbb{R}^{2}\right)$. Because vorticity is transported in 2 d, it remains constant along particle trajectories. If $\Phi_{t}$ is the flow map, then the vorticity is given by $\omega\left(t, \Phi_{t}(y)\right)=\omega(0, y)$, for all $t>0$ and $y \in \mathbb{R}^{2}$. When the initial vorticity is a patch of unit strength, represented by the indicator (characteristic) function $I_{\Omega_{0}}$ of a bounded open set $\Omega_{0} \subset \mathbb{R}^{2}$, the resulting vorticity is $I_{\Omega_{t}}$, with $\Omega_{t}=\Phi_{t}\left(\Omega_{0}\right)$. In the special case when $\Omega_{0}$ is equal to a ball $B$, the patch is stationary, $\Phi_{t}(B)=B$, for all $t>0$. Theorem [3, our main result, gives the stability in $L^{1}\left(\mathbb{R}^{2}\right)$ of any circular patch within the class of all bounded vortex patches of equal strength. No restriction is placed on the $L^{1}$ distance of the perturbation to the ball, and the flow region is not limited to a bounded domain, but rather is the entire space $\mathbb{R}^{2}$.

Wan and Pulvirenti 8 were the first to study stability of vortex patches in $L^{1}$. They considered the case where the flow was contained in a bounded region, although for the stability of circular patches they mention that this assumption can be removed. Their key estimate, $(\mathbf{J})$, shows that the total angular momenta of the patches can be used to control the $L^{1}$ difference between an arbitrary patch and a circular patch of the same total mass. They allow the strengths of the patches to differ, in which case the two patches are assumed to be close in $L^{1}$. Our generalization of their inequality, given in Lemma 2, estimates the $L^{1}$ distance of an arbitrary patch to a circular patch when both patches have equal strength. Stability in $L^{1}$, given in Theorem 13, follows immediately. Weaker stability results were given by Saffman [7] and Dritschel [4. Dritschel controls the measure of the symmetric difference of two patches through a convenient integral, and this idea is incorporated into our argument in Lemma 1 .

Stability in $L^{1}$ does not imply that the boundaries of the two patches remain close in any metric. Indeed, numerical simulations give strong evidence of fingering

Received by the editors April 13, 2009.

2000 Mathematics Subject Classification. Primary 35Q35; Secondary 76B47.

The first author was supported by a grant from the National Science Foundation.

The second author was supported by a grant from the Ministerio de Educación y Ciencia, MTM2007-62186.

The authors thank the anonymous referee for helpful comments.

(C)2009 American Mathematical Society 
and filamentation; see [1, 3]. Spreading of vorticity may also occur. The best upper bound for the growth rate of the patch diameter is $\mathcal{O}(t \log t)^{1 / 4}$ given in [5; see also [6]. Nevertheless, in spite of the fact that the patch geometry may be complicated, smoothness of smooth patch boundaries persists for all time; see [2].

For any bounded open set $A \subset \mathbb{R}^{2}$, denote its mass, momentum, and angular momentum by

$$
|A|=\int_{A} d x, \quad M(A)=\int_{A} x d x, \quad \text { and } \quad i(A)=\int_{A}|x|^{2} d x,
$$

respectively. Our arguments depend heavily upon the fact that these three quantities are conserved in time when $A=\Omega_{t}$ is a patch moving with the flow.

Lemma 1. If $A \subset \mathbb{R}^{2}$ is any bounded open set, then

$$
i(A)-\frac{|A|^{2}}{2 \pi}-\frac{|M(A)|^{2}}{|A|} \geq 0 .
$$

Equality holds if and only if the set $A$ is a ball.

Proof. For any ball $B_{r}\left(x_{0}\right)=\left\{x \in \mathbb{R}^{2}:\left|x-x_{0}\right|<r\right\}$, introduce the quantity

$$
Q=Q\left(A ; B_{r}\left(x_{0}\right)\right)=\int_{A \triangle B_{r}\left(x_{0}\right)}|| x-\left.x_{0}\right|^{2}-r^{2} \mid d x,
$$

in which $A \triangle B_{r}\left(x_{0}\right)=\left(A \backslash B_{r}\left(x_{0}\right)\right) \cup\left(B_{r}\left(x_{0}\right) \backslash A\right)$ denotes the symmetric difference. Note that $Q \geq 0$ and $Q=0$ if and only if $A=B_{r}\left(x_{0}\right)$.

The quantity $Q$ can also be written as

$$
Q=\int_{A}\left(\left|x-x_{0}\right|^{2}-r^{2}\right) d x+\int_{B_{r}\left(x_{0}\right)}\left(r^{2}-\left|x-x_{0}\right|^{2}\right) d x,
$$

since the portions of these two integrals over the set $A \cap B_{r}\left(x_{0}\right)$ cancel each other out.

Now, we can expand the first integral in $Q$ and compute the second to obtain

$$
Q=i(A)-2 x_{0} \cdot M(A)+\left(\left|x_{0}\right|^{2}-r^{2}\right)|A|+\frac{\pi}{2} r^{4} .
$$

A rearrangement of terms gives

$$
Q=i(A)-\frac{|A|^{2}}{2 \pi}-\frac{|M(A)|^{2}}{|A|}+\frac{1}{2 \pi}\left(\pi r^{2}-|A|\right)^{2}+|A|\left|x_{0}-\frac{M(A)}{|A|}\right|^{2} .
$$

This last expression is minimized by choosing $B_{r}\left(x_{0}\right)$ with the same mass and center of mass as $A$ :

$$
\left|B_{r}\left(x_{0}\right)\right|=\pi r^{2}=|A| \quad \text { and } \quad x_{0}=\frac{M(A)}{|A|} .
$$

With this choice, the lemma now follows.

Lemma 2. If $B=B_{r}(0)$, then for any bounded open set $A$,

$$
\left\|I_{A}-I_{B}\right\|_{L^{1}}^{2} \leq 4 \pi Q(A ; B),
$$

in which $Q(A ; B)$ is defined by (11). Equality holds if and only if

$$
A=B_{a}(0) \cup\left[B_{b}(0) \backslash B_{r}(0)\right],
$$

with $a<r<b$ and $r^{2}-a^{2}=b^{2}-r^{2}$. 
Proof. Using the identity (2) and then Lemma 10 we have for any bounded open set $A^{\prime}$,

$$
\left(\left|A^{\prime}\right|-|B|\right)^{2}=\left(\left|A^{\prime}\right|-\pi r^{2}\right)^{2} \leq 2 \pi Q\left(A^{\prime} ; B\right),
$$

with equality if and only if $A^{\prime}$ is a ball centered at the origin.

Next, we note that

$$
\begin{aligned}
\left\|I_{A}-I_{B}\right\|_{L^{1}}^{2} & =|A \Delta B|^{2} \\
& =(|A \backslash B|+|B \backslash A|)^{2} \\
& \leq 2|A \backslash B|^{2}+2|B \backslash A|^{2} \\
& =2(|A \cup B|-|B|)^{2}+2(|A \cap B|-|B|)^{2},
\end{aligned}
$$

with equality if and only if $|A \backslash B|=|B \backslash A|$.

Application of (4) with $A^{\prime}=A \cup B$ and $A^{\prime}=A \cap B$ yields

$$
\begin{aligned}
2(|A \cup B|-|B|)^{2}+2(|A \cap B|-|B|)^{2} & \\
& \leq 4 \pi[Q(A \cup B ; B)+Q(A \cap B ; B)]=4 \pi Q(A ; B),
\end{aligned}
$$

with equality if and only if $A \cup B$ and $A \cap B$ are balls centered at the origin. This establishes the desired inequality.

The argument also shows that equality holds if and only if $A \cup B=B_{b}(0)$, $A \cap B=B_{a}(0)$, with $a<r<b$, and

$$
\left|B_{b}(0) \backslash B\right|=|A \backslash B|=|B \backslash A|=\left|B \backslash B_{a}(0)\right|,
$$

which gives (3).

Theorem 3. Let $B=B_{r}(0)$. Then for any bounded open set $\Omega_{0} \subset \mathbb{R}^{2}$, we have that

$$
\left\|I_{\Omega_{t}}-I_{B}\right\|_{L^{1}}^{2} \leq\left. 4 \pi \sup _{\Omega_{0} \triangle B}|| x\right|^{2}-r^{2} \mid\left\|I_{\Omega_{0}}-I_{B}\right\|_{L^{1}}
$$

for all $t>0$.

Proof. The identity (2) shows that the quantity $Q\left(\Omega_{t} ; B\right)$ depends only on conserved quantities, and it is therefore also conserved. In other words, we have $Q\left(\Omega_{t} ; B\right)=$ $Q\left(\Omega_{0} ; B\right)$, for all $t>0$. Thus, the result follows from Lemma 2 and the fact that

$$
Q\left(\Omega_{0} ; B\right) \leq\left.\sup _{\Omega_{0} \triangle B}|| x\right|^{2}-r^{2}|| \Omega_{0} \triangle B\left|=\sup _{\Omega_{0} \triangle B}\right||x|^{2}-r^{2} \mid\left\|I_{\Omega_{0}}-I_{B}\right\|_{L^{1}} .
$$

\section{REFERENCES}

[1] Buttke, T.F. A fast adaptive vortex method for patches of constant vorticity in two dimensions. J. Comput. Phys. 89 (1990), no. 1, 161-186. MR.1063151 (91g:76072)

[2] Chemin, J.-Y. Sur le mouvement des particules d'un fluide parfait incompressible bidimensionnel. Invent. Math. 103 (1991), 599-629. MR1091620 (91m:35187)

[3] Deem, G.S. and N.J. Zabusky. Vortex waves: stationary "V states", interactions, recurrence, and breaking. Phys. Rev. Lett. 41 (1978), no. 13, 859-862.

[4] Dritschel, D.G. Nonlinear stability bounds for inviscid, two-dimensional, parallel or circular flows with monotonic vorticity, and the analogous three-dimensional quasi-geostrophic flows. J. Fluid Mech. 191 (1988), 575-581.

[5] Iftimie, D., T.C. Sideris, and P. Gamblin. On the evolution of compactly supported planar vorticity. Comm. Partial Differential Equations 24 (1999), no. 9-10, 1709-1730. MR.1708106 (2000d:76030) 
[6] Marchioro, C. Bounds on the growth of the support of a vortex patch. Comm. Math. Phys. 164 (1994), 507-524. MR 1291243 (95f:76012)

[7] Saffman, P.G. Vortex dynamics. Cambridge Monographs on Mechanics and Applied Mathematics. Cambridge University Press, New York, 1992. MR.1217252 (94c:76015)

[8] Wan, Y.H. and M. Pulvirenti. Nonlinear stability of circular vortex patches. Comm. Math. Phys. 99 (1985), no. 3, 435-450. MR795112 (86i:76028)

[9] Yudovič, V.I. Non-stationary flow of an ideal incompressible liquid. Z̆. Vyčisl. Mat. i Mat. Fiz. 3 (1963), no. 6, 1032-1066. MR0158189 (28:1415)

Department of Mathematics, University of California, Santa Barbara, California 93106

E-mail address: sideris@math.ucsb.edu

Departamento de Matemáticas, Universidad del País Vasco, Apartado 644, 48080 BilBAO, SPAIN

E-mail address: luis.vega@ehu.es 